\title{
Commentary: Association between Poor Sleep Quality and Subsequent Peptic Ulcer Recurrence in Older Patients with Mild Cognitive Impairment: Examining the Role of Social Engagement
}

\author{
Wenni Chen', Yewei Bao', Yu Yu', Ruirui Xu', Shuyan Yang'2, Hui Han², Gengzhen Chen* \\ 'Shantou University Medical College, Shantou, China \\ ${ }^{2}$ Hong Kong Baptist University, Kowloon Tong, Hong Kong, China
}

\section{Article Info}

\section{Article Notes}

Received: April 21, 2019

Accepted: May 27, 2019

\section{${ }^{*}$ Correspondence:}

Dr. Gengzhen Chen, Shantou University Medical College,

Shantou, China; Email: gzchen12@stu.edu.cn or chengengzhen@126.com.

(c) 2019 Chen $\mathrm{G}$. This article is distributed under the terms of the Creative Commons Attribution 4.0 International License.

\section{Keywords}

Association

Sleep quality

PUD recurrence

Social engagement

Older patient

H. pylori.
Poor sleep quality is one of the most crucial problems impairing the health of the elderly both physically and psychologically, and so is peptic ulcer disease (PUD). Current findings have exposed us to the potential pathways of recurrent PUD except for H. Pylorireinfection ${ }^{1}$. At the same time, it has been proved that poor sleep quality may damage digestive health by disturbing its protective system $^{2}$. In another cross-sectional study conducted by Wang et al., chronic gastroenteritis and peptic ulcer was ascribed to short sleep duration ${ }^{3}$. However, as the authors pointed out, there isn't any existing study confirming the association between poor sleep quality and PUD recurrence and the role of social engagement in that relation. Moreover, the limitations of other studies rest with their cross-sectional designs and retrospective data. Hence, they conducted a 3-year longitudinal study to settle problems above.

Three years of data from eight Grade-A hospitals have been reviewed in this research by Fang et al. Briefly, the authors evaluated 1689 patients catching both PUD secondary to H. Pylori-infection and mild cognitive impairment, and recoveries of ulcers followed successful H. Pylori eradication for 1538 (91.1\%) of them. Then they measured post-eradication variables including relapse of PUD, self-perceived sleep quality and variations in social engagement. According to their sleep quality and social engagement conditions, five groups were made including Group 1 (with good sleep quality), Group 2 (with poor sleep quality and increased social engagement), Group 3 (with poor quality and continued social engagement), Group 4 (with poor sleep quality and decreased social engagement), and Group 5 (with poor sleep quality but without social engagement). Thirty-six months of follow-up was carried out, excluding those receiving ulcer-related and/or sleep interfering drugs, digestive tract operations and any other missing out with 1420 left.

Out of 1420 patients, 118 (8.2\%) of them were found to have recurrent PUD within 36 months and higher recurrence rate $(10.8 \%)$ was observed in those with poor sleep quality than that in those with good sleep quality (5.5\%). When adjusted for other confounders, it was shown that poor sleep quality raised the recurrence of PUD in multivariate analysis. Whereas socioeconomic status, cardiovascular disease, depression and H. pylori reinfection at follow-up also exhibited associations with subsequent PUD relapse in the meantime. In addition, significant interactions have been revealed between social engagement changes and poor sleep 
quality. Also, in additional multivariate analysis, a higher risk of PUD recurrence was demonstrated in Group 4 and Group 5 as compared with Group 1, but no difference was seen between Group 1, Group 2 and Group 3.

This study advanced previous research on the field of sleep and PUD by using a longitudinal design to identify the specific link between poor sleep quality and recurrent PUD, supporting previous studies that proposed the possible trend of poor sleep quality generating PUD relapse ${ }^{4}$. Furthermore, these findings offer a constructive and insightful suggestion to physicians highlighting the worth of addressing sleep disorders in patients after $H$. Pylori eradication, especially for those with reinfection. Yet the implementation of new clinical protocols needs more valid clinical evidences in which treatments of poor sleep quality and management of social engagement would be utilized to prevent PUD relapse.

Former studies did make it clear that increased social engagement may improve the physical and mental health of older people ${ }^{5,6}$. Meaningfully, Chen et al. broadened our view in revealing the potential impact of social engagement to block PUD recurrence by interfering in the sleep state of old people. Thus, it is also important to recommend attending social activities to alleviate poor sleep quality and subsequently guard against possible relapse of ulcers.

In this study, however, not all groups of patients with poor sleep quality tended to avoid ulcer relapse in spite of increased social engagement. Perhaps something deep-going would be interpreted with the more reasonable classification of patients, more scientific ways of measurements, and initiative management of social engagement. Besides, that provokes our thinking on the detailed mechanism of social engagement as a mediator in the association between poor sleep quality and PUD recurrence as well. From another angle, the exploration of the role of social engagement not merely paved ways for future researches on this subject in study design, but opened a door to the underlying mechanism researches around it.

It is valuable to find out the authors self-reflected limitations of this study without concealing them at all. One of them noteworthy was the "bi-directionality" between PUD symptoms and poor sleep quality. Of course, it's hard to tell whether poor sleep quality led to recurrent PUD or the other way around and there doesn't exist any perfect study design. However, in my eyes that isn't an unnecessary talk but a critical way of thinking benefiting other researchers of upcoming studies.

On the other hand, although the authors had indicated that one of the limitations of their studies lied in the "selfreporting approach" to determine their sleep quality, which implies the shortage of objective measurements, they resolved this issue in another study by Chen et al. published on Journal of the American Geriatrics Society ${ }^{z}$. They assessed objective sleep quality using a multisensory body monitor in the later study and observed longer total sleep time lowered the rate of PUD recurrence. Unprecedentedly, they proved the higher risk of ulcer relapse stemmed from longer sleep onset latency and more nighttime awakenings. Up to now, the team of Dr. Chen has demonstrated both subjective and objective poor sleep quality predicts recurrent PUD in older patients which is definitely a pioneering work.

Is there something more in-depth we can conclude from this essay? We cannot underrate the significance of psychiatry and sleep medicine in treating physical diseases such as PUD, and this point was reinforced in this article because depressive symptoms and generalized anxiety disorders also showed influence on PUD recurrence. Apart from PUD, other chronic disease as well as mental disorders has been confirmed to be associated with abnormal sleep durations ${ }^{3}$. And that requires the collaborations between physicians and psychiatrists. What is more, since social engagement might be conducive to older patients in relapse prevention, discharge instruction and health education shouldn't be neglected. Then it is worth stressing the cooperation with nurses in charge of those works. It's not hard to see the weight of multidisciplinary collaboration in the prevention and treatment of diseases from this study.

\section{References}

1. Tseng GY, Lin HJ, Fang CT, et al. Recurrence of peptic ulcer in uraemic and non-uraemic patients after Helicobacter pylori eradication: a 2-year study. Aliment Pharmacol Ther. 2007; 26(6): 925-33.

2. Khanijow V, Prakash P, Emsellem HA, et al. Sleep Dysfunction and Gastrointestinal Diseases. Gastroenterol Hepatol (N Y). 2015; 11(12): 817-25.

3. Wang $\mathrm{S}, \mathrm{Wu} \mathrm{Y}$, Ungvari GS, et al. Sleep duration and its association with demographics, lifestyle factors, poor mental health and chronic diseases in older Chinese adults. Psychiatry Res. 2017; 257: 212-218.

4. Räihä M, Seppälä 0 , Impivaara MT, et al. Chronic illness and subjective quality of sleep in the elderly. Aging (Milano). 1994; 6(2): 91-6.

5. Park NS. The Relationship of Social Engagement to Psychological Well-Being of Older Adults in Assisted Living Facilities. J Appl Gerontol. 2009; 28(4): 461-481.

6. Thoits PA. Mechanisms linking social ties and support to physical and mental health. J Health Soc Behav. 2011; 52(2): 145-61.

7. Fang B, Liu H, Yang S, et al. Effect of Subjective and Objective Sleep Quality on Subsequent Peptic Ulcer Recurrence in Older Adults. J Am Geriatr Soc. 2019; [Epub ahead of print]. 